\title{
Carbonatación de pastas de cemento de aluminato de calcio
}

\section{Carbonation of calcium aluminate cement pastes}

\author{
L.FERNÁNDEZ-CARRASCO,F.PUERTAS,M.T. BLANCO-VARELA,T.VÁZQUEZ
}

Instituto de Ciencias de la Construcción Eduardo Torroja (CSIC)

Fechaderecepción:2-VII-2001

ESPAÑA

\section{RESUMEN}

En el presente trabajo se discuten los resultados obtenidos en los ensayos acelerados llevados a cabo para investigar los efectos de diferentes métodos de curado sobre la carbonatación de pastas del cemento de aluminato de calcio (CAC). Se estudió la composición mineralógica de las muestras hidratadas y carbonatadas. Además, se determinaron las resistencias mecánicas a compresión y la porosidad de las probetas.

Los resultados indican que la vaterita y el aragonito son las polimorfias del $\mathrm{CaCO}_{3}$ que se forman al carbonatar los aluminatos cálcicos hidratos, tanto los de naturaleza hexagonal como cúbica. El polimorfo del carbonato cálcico formado no depende del proceso de curado. La velocidad de carbonatación de los hidratos hexagonales es mayor que la de los cúbicos. Los resultados obtenidos en el presente trabajo han evidenciado que como consecuencia del proceso de carbonatación sobre pastas de CAC, en las condiciones realizadas, se produce un incremento en las resistencias mecánicas.

\section{SUMMARY}

This work discusses the results from accelerated tests intended to investigate the ways the different curing methods affect the carbonation of calcium aluminate cements pastes (CAC). The research was focused on the mineralogical composition of hydrated and carbonated samples. The compressive strengths and the porosity of the samples have been determined.

Results point out that vaterite and aragonite are formed as a result of carbonation of both cubic and hexagonal calcium aluminate hydrates. The polymorph of calcium carbonate formed does not depend on the curing process. Carbonation rates is higher in hexagonal than in cubic hydrates. Results obtained through this study evidence that, as a consequence of the carbonation process of $C A C$ pastes, in test conditions, an increase of the mechanical strengths occurs.

\section{INTRODUCCIÓN}

La carbonatación de las pastas de cemento portland es un fenómeno que ha sido ampliamente estudiado; no ocurre lo mismo en el caso de las pastas de cemento de aluminato de cálcico (CAC). Como consecuencia de una serie de incidentes que sucedieron en España y que se asociaron, entre otras causas, a efectos de la carbonatación del CAC (1) se puso de manifiesto la necesidad de ampliar la investigación sobre el tema y de forma específica sobre el fenómeno denominado "hidrólisis alcalina", que corresponde a un tipo de carbonatación en presencia de álcalis.

\section{INTRODUCTION}

Carbonation of Portland cement pastes is a phenomenon that has been widely studied; whereas the case of calcium aluminate cement pastes (CAC) has not. Consequently, after a series of incidents that happened in Spain, which were meant to be due, among other agents, to carbonation effects of CAC (1) brought awareness on the need to deepen the research on this subject and specifically on the phenomenon called "alkaline hydrolysis", which corresponds to a type of carbonation in presence of alkalis. 
Con respecto a la carbonatación en ausencia de álcalis, lamentablemente, los trabajos existentes muestran con frecuencia resultados contradictorios, mientras que los referentes a la carbonatación en presencia de álcalis eran meramente teóricos, hasta que recientemente dicho proceso ha podido reproducirse en laboratorio (2).

Se ha comprobado que hay un gran número de variables que afectan, tanto al proceso de hidratación como al de carbonatación del CAC (2), tales como la temperatura, el tiempo, la relación agua/cemento, el tipo de curado, la concentración de $\mathrm{CO}_{2}$ y la humedad relativa. Además, en el caso de morteros y hormigones también tienen influencia en esos procesos el tipo y naturaleza de la arena y/o áridos utilizados. Pequeñas variaciones en dichos parámetros conllevan grandes modificaciones en los resultados. Debido a que el "modus operandi" de los distintos grupos investigadores generalmente es diferente, gran parte de los trabajos que existen en la bibliografia, aunque de gran interés, son difícilmente comparables (3-7). En el estudio de los procesos de hidratación y de carbonatación del CAC es de considerable importancia, por tanto, establecer con gran rigor las variables iniciales de los procesos a estudiar.

El objetivo planteado en el presente trabajo ha sido conocer el efecto de diferentes métodos de curado sobre la carbonatación de pastas del cemento de aluminato de calcio.

\section{EXPERIMENTAL}

Se prepararon probetas prismáticas de pastas de CAC de dimensiones $1 \times 1 \times 6 \mathrm{~cm}$. Para la preparación de estas probetas se amasó el CAC con agua destilada y desionizada. Las temperaturas de amasado empleadas fueron $4^{\circ}$ y $40^{\circ} \mathrm{C}$ mientras que la relación agua/ cemento fue de 0,8 . Para cada temperatura de amasado se hicieron dos series de probetas. Una de las series fue curada durante 14 días mientras que la otra se curó durante 43 días. Ambas series permanecieron inicialmente 7 días al $100 \%$ de H.R. y a continuación se mantuvieron al $75 \%$ hasta completar el tiempo prefijado. Se emplea la letra "F" para designar las muestras elaboradas a la temperatura de $4^{\circ} \mathrm{C}$ mientras que la "C" indica las preparadas a $40^{\circ} \mathrm{C}$. El subíndice utilizado señala el tiempo de curado de las probetas. Un esquema de los diferentes ensayos realizados se presenta en la Fig. 1

Las probetas se sometieron, tras el curado, a un proceso de carbonatación acelerado. Dicho proceso tuvo lugar en un desecador de 25 litros de capacidad, que fue utilizado a modo de cámara de carbonatación.
Unfortunately works available on carbonation in absence of alkalis, often show contradictory results, while those referring to carbonation in presence of alkali were merely theoretical until it has been possible to reproduce the process in the laboratory (2).

It has been proved that a substantial number of variables affect the CAC hydration and carbonation processes: temperature, time, water/cement ratio, the kind of curing conditions, $\mathrm{CO}_{2}$ concentration, and relative humidity (2). Moreover, in the case of mortars and concretes, the kind and nature of sand and/or the aggregates used also influences these processes. Slight variations in such parameters can modify results severely. Considering the differentiate "modus operandi" of the several research groups, most part of the works appearing in bibliography, although being very interesting, can hardly be contrasted one another (3-7). Therefore, in the study and research of hydration and carbonation processes of the CAC it is very important to establish carefully the initial variables of the processes to be studied.

The main objective that has been considered in this work is to know the effect of different curing methods on carbonation of calcium aluminate cement pastes.

\section{EXPERIMENTAL}

Prismatic samples sized $1 \times 1 \times 6 \mathrm{~cm}$ were prepared with CAC pastes. For their preparation, CAC was mixed with distilled and de-ionized water. Mixing temperatures were $4^{\circ}$ and $40^{\circ} \mathrm{C}$ and cement/water ratio was 0.8 . For each mixing temperature two series of test samples were made. One of the series was cured for 14 days while the other was cured for 43 days. Initially both series were kept during 7 days at $100 \%$ R.H. and thereafter at $75 \%$ until the preestablished time was completed. An " $F$ " is used to identify samples made at $4{ }^{\circ} \mathrm{C}$ temperature while " $C$ " particularizes those prepared at $40{ }^{\circ} \mathrm{C}$. The sub-index indicates the curing time of the samples. An outline of the different tests carried out is shown in Fig. 1 .

Once the curing time was over, samples were submitted to an accelerated process of carbonation. Such process took place in a 25-liters capacity carbonation chamber. Environmental conditions 


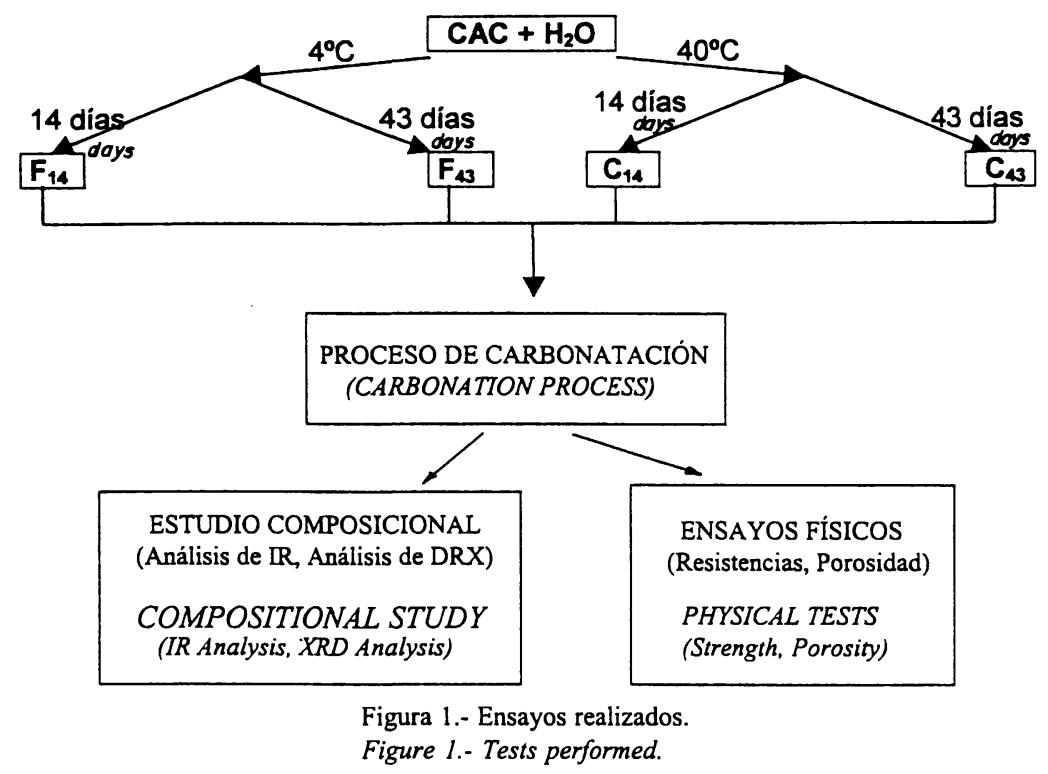

Las condiciones ambientales fijadas en la cámara fueron: humedad relativa del $75 \%$, temperatura de 20 ${ }^{\circ} \mathrm{C}$ y ambiente saturado de $\mathrm{CO}_{2}$.

Las probetas se caracterizaron mediante Difracción de Rayos X (DRX) y Espectroscopia de Infrarrojo (FTIR). Se examinó la parte externa de las probetas carbonatadas a 3, 7, 14, 28 y 43 días a través FTIR. Se analizó por ambas técnicas, DRX y FTIR, a 56 y 180 días de carbonatación tanto la parte externa como la interna.

Se determinaron las resistencias a compresión de las probetas. Los valores se obtuvieron a partir de la media de los resultados de 12 probetas de cada edad. A través de ensayos de porosimetría de mercurio se determinó la porosidad y la distribución del tamaño de poro de las muestras.

\section{RESULTADOS}

En la Tabla I se presentan los compuestos de hidratación y carbonatación de naturaleza cristalina detectados al analizar las muestras mediante DRX. Se realizó una estimación semicuantitativa de la proporción relativa de los compuestos formados en cada una de las muestras.

Del análisis de esta Tabla se deduce que las pastas $\mathrm{F}$ (que contienen los aluminatos cálcicos hidratados hexagonales) se carbonatan a mayor velocidad que las pastas $\mathrm{C}$ (aluminatos cálcicos hidratados cúbicos), $\mathrm{y}$ que el tiempo de curado influye acelerando dicho proceso. Los principales compuestos carbonatados en las diferentes pastas son aragonito y vaterita. La proporción de estos es mayor en las pastas preparadas a menor temperatura. $\mathrm{El} \mathrm{CaCO}_{3}$, calcita, únicamente es detectado en algunas pastas a nivel de trazas. $\mathrm{El} \mathrm{AH}_{3}$ es within the chamber were: $20^{\circ} \mathrm{C}, 75 \%$ relative humidity and an environment saturated with $\mathrm{CO}_{2}$.

Samples were characterized through $X$ Ray Diffraction (XRD) and Infrared Spectroscopy (FTIR). The outer layer of the carbonated samples was studied through FTIR after 3, 7, 28, 14 and 43 days. Those samples carbonated for 56 and 180 days were analyzed through both XRD and FTIR and not only the outer layer but also the interior.

Compression strengths of prisms were determined. Values were reckoned taking the average results from 12 samples of each age. Through mercury porosimetry tests, porosity of the samples and pore size distribution were also determined.

\section{RESULTS}

Table I shows the crystalline hydrated and carbonated compounds detected when analyzing test samples through XRD. A semi-quantitative estimate of the amount of compounds formed was done for every one of the samples.

From the analysis of this Table it is inferred that $F$ pastes (which contain hexagonal calcium aluminate hydrates) develop carbonation at higher rate than $C$ pastes (calcium aluminate cubic hydrates), and that samples cured for more time carbonate more quickly. The main carbonated compounds among the different pastes are aragonite and vaterite. The proportion of these compounds is higher in pastes prepared at lower temperature. Only traces of calcite are detected in some pastes. The $\mathrm{AH}_{3}$ is 
TABLA I/TABLEI

Compuestos detectados por DRX / (Compounds detected through XRD)

\begin{tabular}{|c|c|c|c|c|c|c|c|c|c|c|}
\hline & \multicolumn{2}{|c|}{ Tiempo / Time } & $\mathrm{CAH}_{10}$ & $\mathrm{C}_{2} \mathrm{AH}_{8}$ & $\mathrm{C}_{3} \mathrm{AH}_{6}$ & $\mathrm{AH}_{3}$ & MCA & $\begin{array}{l}\text { CALCITA } \\
\text { CALCITE }\end{array}$ & $\begin{array}{l}\text { ARAGONITO } \\
\text { ARAGONITE }\end{array}$ & $\begin{array}{l}\text { VATERITA } \\
\text { VATERITE }\end{array}$ \\
\hline \multirow{5}{*}{$F_{14}$} & \multicolumn{2}{|c|}{$\operatorname{Sin} I$ Without $\mathrm{CO}_{2}$} & 3 & $1(\alpha)$ & - & - & * & - & - & - \\
\hline & \multirow{2}{*}{$\begin{array}{c}2 \text { meses } \\
2 \text { months } \\
\mathrm{CO}_{2}\end{array}$} & Intern. & 1 & - & - & $*$ & - & $*$ & 1 & 2 \\
\hline & & Extern. & 1 & - & - & * & - & * & 1 & 2 \\
\hline & \multirow{2}{*}{$\begin{array}{c}6 \text { meses/ } \\
6 \text { months } \\
\mathrm{CO}_{2}\end{array}$} & Intern. & $*$ & - & - & * & - & * & 2 & 3 \\
\hline & & Extern. & $*$ & - & - & * & - & $*$ & 2 & 3 \\
\hline \multirow{5}{*}{$F_{43}$} & \multicolumn{2}{|c|}{ Sin I Without $\mathrm{CO}_{2}$} & 3 & $1(\alpha)$ & - & - & - & - & - & - \\
\hline & \multirow{2}{*}{$\begin{array}{c}2 \text { meses } \\
2 \text { months } \\
\mathrm{CO}_{2}\end{array}$} & Intern. & 1 & - & - & - & - & - & 1,5 & 2 \\
\hline & & Extern. & 0,5 & - & - & - & - & - & 1 & 2,5 \\
\hline & \multirow{2}{*}{$\begin{array}{c}6 \text { meses } \\
6 \text { months } \\
\mathrm{CO}_{2}\end{array}$} & Intern. & $*$ & - & - & $\mathbf{n}$ & - & - & 1,5 & 2 \\
\hline & & Extern. & $*$ & - & - & b & - & - & 2 & 1,5 \\
\hline \multirow{5}{*}{$\mathrm{C}_{14}$} & \multicolumn{2}{|c|}{ Sin I Without $\mathrm{CO}_{2}$} & - & $1(\beta)$ & 4 & 1 & * & - & -- & - \\
\hline & \multirow{2}{*}{$\begin{array}{c}2 \text { meses } \\
2 \text { months } \\
\mathrm{CO}_{2}\end{array}$} & Intern. & - & - & 3 & 1 & - & $*$ & * & 1 \\
\hline & & Extern. & - & - & 3 & 1 & - & - & * & 1 \\
\hline & \multirow{2}{*}{$\begin{array}{c}6 \text { mesesl } \\
6 \text { months } \\
\mathrm{CO}_{2}\end{array}$} & Intern. & - & - & 3 & $\mathbf{g}, \mathbf{n}$ & - & * & 1 & 1 \\
\hline & & Extern. & - & - & 3 & $\mathbf{g}, \mathbf{n}$ & - & - & 1 & 1 \\
\hline \multirow{5}{*}{$\mathrm{C}_{43}$} & \multicolumn{2}{|c|}{ Sin I Without $\mathrm{CO}_{2}$} & - & $*(\beta)$ & 4 & $*$ & - & - & - & - \\
\hline & \multirow{2}{*}{$\begin{array}{c}2 \text { meses } \\
2 \text { months } \\
\mathrm{CO}_{2}\end{array}$} & Intern. & - & - & 3,5 & $*$ & - & * & 1 & * \\
\hline & & Extern. & - & - & 3 & * & - & * & 1 & $*$ \\
\hline & \multirow{2}{*}{$\begin{array}{c}6 \text { mesesl } \\
6 \text { months } \\
\mathrm{CO}_{2}\end{array}$} & Intern. & - & - & 2,5 & 1 & - & * & 1 & * \\
\hline & & Extern. & - & - & 2 & 1 & - & * & 1 & * \\
\hline
\end{tabular}

Maximun Intensity: 4, traces: * n: nordstrandite, b: bayerite, g: gibbsite , mCA: $\mathrm{C}_{4} A c H_{11}$ Intensidad máxima: 4, trazas: *. n: nordstrandita, b: bayerita, g: gibbsita, mCA: $\mathrm{C}_{4} \mathrm{ACH}$

identificado en sus tres formas polimórficas, aunque preferentemente en las muestras $\mathrm{F}_{43}$ como nordstrandita y bayerita y en las $\mathrm{C}_{14}$ como nordstrandita y gibbsita. La proporción de estas fases identificadas por DRX es muy pequeña, siendo mayor en las pastas $C$ que en las $F$.

La evolución de la carbonatación fue también seguida mediante FTIR (Fig. 2). Se observa en los espectros IR de las muestras $\mathrm{F}_{14} \mathrm{y} \mathrm{F}_{43}$, un ensanchamiento $\mathrm{y}$ asimetría de las dos ramas de la banda debida a vibraciones de tensión de los grupos $\mathrm{OH}$, a medida que se incrementa el tiempo de carbonatación. Ello se interpreta que es causado por la formación de $\mathrm{AH}_{3}$ simultáneamente a la desaparición de los aluminatos cálcicos hidratados. No obstante la presencia, en la región de 3400-3700 $\mathrm{cm}^{-1}$, de una banda aparentemente única, ancha y sin otras bandas de absorción estrechas y bien definidas indicaría que se trata de $\mathrm{AH}_{3}$ de naturaleza amorfa. En lo que respecta al $\mathrm{CaCO}_{3}$ formado, los espectros presentan bandas de absorción debidas fundamentalmente a la vaterita y al aragonito. identified in its three polymorphs, although mainly in $F_{43}$ samples as nordstrandite and bayerite and in the $C_{14}$ samples as nordstrandite and gibbsite. The amount of these phases identified through XRD is very small, being higher in $C$ than in $F$ pastes.

Evolution of carbonation was followed by FTIR (Fig. 2). IR spectra of samples $F_{14}$ and $F_{43^{3}}$ an enlargement and an asymmetry of the two branches of the band, due to stretching vibrations of $\mathrm{OH}$ groups, as carbonation time increased. The cause could be the formation of $\mathrm{AH}_{3}$, which was developing at the same rate the hydrated calcium aluminates were disappearing. However, the presence in region $3400-3700 \mathrm{~cm}^{-1}$ of a band apparently unique, wide and without any other narrow and well-defined absorption band, would suggest the presence of amorphous $\mathrm{AH}_{3}$. As formed $\mathrm{CaCO}_{3}$, spectra showed absorption bands mainly due to vaterite and aragonite. 
En los espectros de IR de las muestras $\mathrm{C}_{14}$ y $\mathrm{C}_{43}$ (Fig. 2) se deduce que con el tiempo de exposición al $\mathrm{CO}_{2}$ disminuyen las bandas debidas al compuesto $\mathrm{C}_{3} \mathrm{AH}_{6}$. $\mathrm{A}$ través de las variaciones de la banda más característica de dicho compuesto, en $3660 \mathrm{~cm}^{-1}$; se observa que es más efectiva la carbonatación en la parte externa de la probeta. Dado que las muestras sin carbonatar ya contienen gran cantidad de $\mathrm{AH}_{3}$, mediante esta técnica es dificil establecer conclusiones sobre el tipo de $\mathrm{AH}_{3}$ formado como consecuencia del proceso de

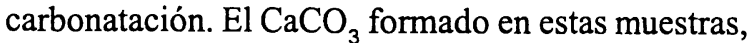
tanto las curadas 14 como 43 días, está en forma de vaterita y de aragonito.
From IR spectra of samples $C_{14}$ and $C_{43}$ (Fig. 2) it is inferred that the longer the exposure time to $\mathrm{CO}_{2}$ is, the more the bands corresponding to $C_{3} A H_{6}$ decrease. Through the variations of the most characteristic band of that compound, at $3660 \mathrm{~cm}^{-1}$, it is observed that carbonation is more effective in the external layer of the sample. Since samples bearing no carbonation already contained large amounts of $\mathrm{AH}_{3}$, it is difficult to establish conclusions through this technique on the type of $\mathrm{AH}_{3}$ formed as a consequence of the carbonation process. The $\mathrm{CaCO}_{3}$ developed in samples cured at 14 days, as much as those cured at 43 days, appeared as vaterite and aragonite.

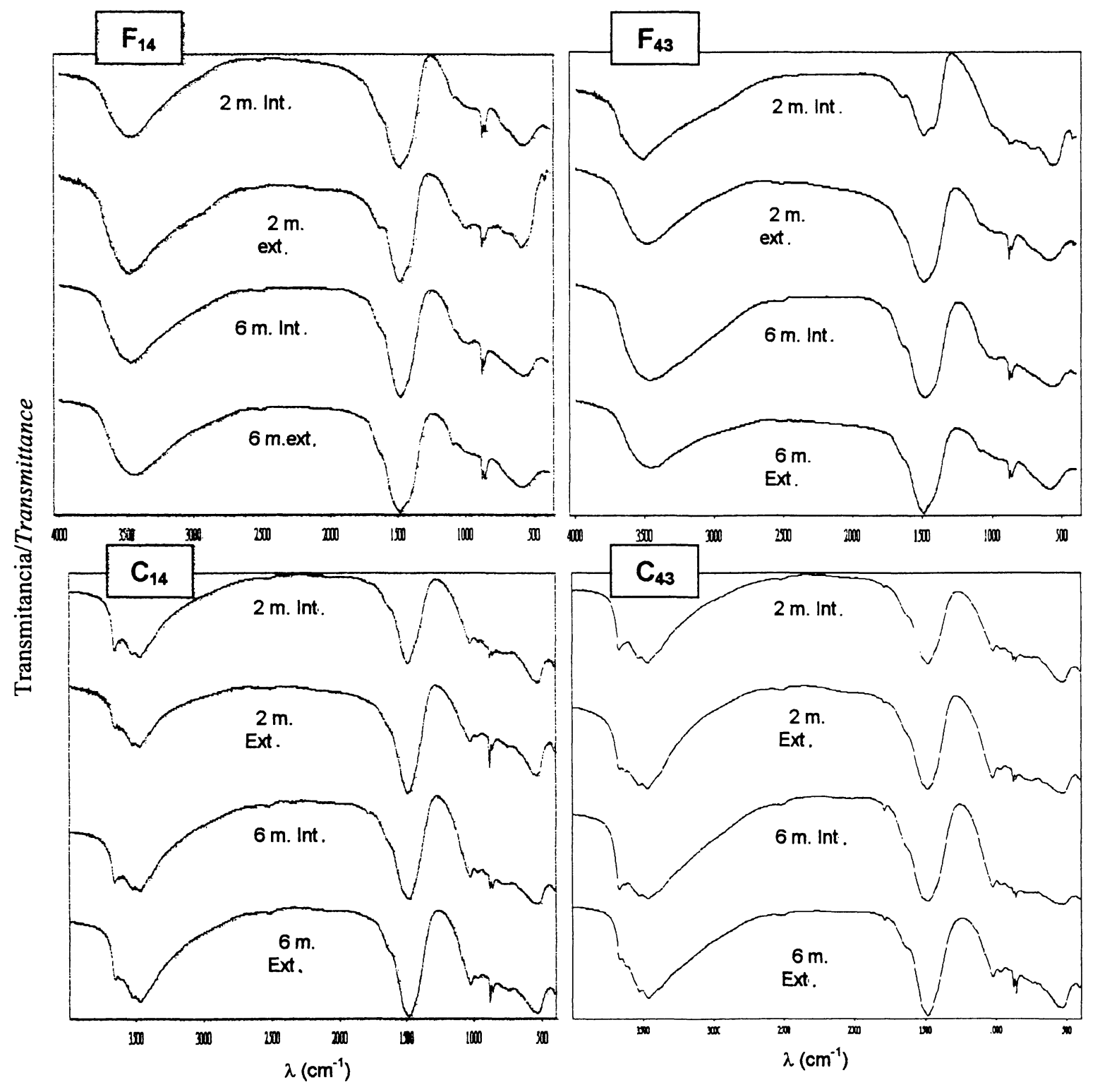

Figura 2.- Espectros de IR de las muestras $\mathrm{F}_{14}, \mathrm{~F}_{43}, \mathrm{C}_{14}$ y $\mathrm{C}_{43}$.

Figure 2.- IR spectra of samples $F_{14} F_{43} C_{14}$ and $C_{45}$ 
Los resultados obtenidos en los ensayos realizados de porosidad total y resistencias mecánicas a compresión se muestran en la Tabla II. Se ha podido comprobar que a los dos meses de acción del $\mathrm{CO}_{2}$ las probetas sufrieron un considerable descenso de la porosidad total y un incremento de las resistencias mecánicas. La carbonatación de las muestras, tanto las tipo F como las $\mathrm{C}$, produce un efecto muy positivo sobre la resistencia a compresión; no obstante, esa resistencia es considerablemente superior en las probetas tipo $\mathrm{F}$ a la mostrada por las de tipo C.

Las variaciones en la distribución del tamaño de poros se presentan en la Fig. 3. Se puede observar que las probetas tipo $\mathrm{F}$ no expuestas a la acción del $\mathrm{CO}_{2}$. contienen una red porosa en la que prácticamente el $60 \%$ de esos poros tiene un tamaño comprendido entre 0,1 y $1 \mu \mathrm{m}$. En las probetas que han estado expuestas al $\mathrm{CO}_{2}$ durante 2 meses, los poros de ese rango descienden en un $35 \% \mathrm{y}$, por el contrario, los de un tamaño comprendido entre 0,01 y $0,1 \mu \mathrm{m}$ se incrementan en un $35 \%$. Los poros de tamaño comprendido entre $1-10 \mu \mathrm{m}$ disminuyen en un $10 \% \mathrm{y}$ recíprocamente aumentan en ese mismo orden (10\%) los inferiores a $0,01 \mu \mathrm{m}$. La distribución de poros en las probetas tratadas con $\mathrm{CO}_{2}$ durante seis meses no experimentan una evolución significativa, respecto a la descrita en las de dos meses.

Las probetas tipo $\mathrm{C}$, previo a su carbonatación, presentan un $80 \%$ de sus poros con tamaño comprendido entre 1 y $10 \mu \mathrm{m}$, independientemente del tiempo de curado. Transcurridos dos meses de carbonatación el porcentaje de esos poros disminuye hasta el $45 \%$, mientras que aumentan en un $20 \%$ los comprendidos entre 0,01 y $0,1 \mu \mathrm{m}$ y en un $15 \%$ los de $0,1-1 \mu \mathrm{m}$. No se altera la red porosa entre los dos y los seis meses de carbonatación (ver Fig.3).
Results obtained from tests on total porosity and mechanical compressive strengths are shown in Table II. It has been possible to prove that after two months under the $\mathrm{CO}_{2}$ action, samples undergo a remarkable decrease of total porosity and an increase of mechanical strengths. Carbonation of samples of both types, $F$ and $C$, act positively under compressive strength. However, this strength is considerably higher in $F$ rather than $C$ samples.

Variations of pore size distribution are shown in Figure 3. This figure points out that $F$ samples, which have not been exposed to $\mathrm{CO}_{2}$ action, hold a porous network where practically $60 \%$ of pores are sized 0.1 to $1 \mu \mathrm{m}$. In samples that have been exposed to $\mathrm{CO}_{2}$ for two months, pores within this range decrease $35 \%$, as opposed to those ranging from 0.01 to $0.1 \mu \mathrm{m}$, which bear an increase of $35 \%$. Pores within size 1 to $10 \mu \mathrm{m}$ decrease $10 \%$ and reciprocally pores smaller than $0.01 \mu \mathrm{m}$ augment in the same proportion (10\%). Size distribution pore of samples treated with $\mathrm{CO}_{2}$ for six months do not bear a remarkable evolution compared to those treated for two months.

Before carbonation, samples $C$ have $80 \%$ of the pores ranging from 1 to $10 \mathrm{~m}$, independently of the curing time. After two carbonation months the percentage of these pores decreases until $45 \%$, while those between 0.01 and $0.1 \mu \mathrm{m}$ augments $20 \%$, and $15 \%$ those between $0.1-1 \mu \mathrm{m}$. The pore structure does not bear any alteration between two and six months carbonation (see Fig. 3).

TABLA II / TABLE II

Porosidad y resistencia / (Porosity and strength)

\begin{tabular}{|c|c|c|c|c|}
\hline & & $\begin{array}{c}\text { SIN I } \\
\text { WITHOUT } \mathrm{CO}_{2}\end{array}$ & $2 \mathrm{~m} \cdot \mathrm{CO}_{2}$ & $6 \mathrm{~m} . \mathrm{CO}_{2}$ \\
\hline \multirow{4}{*}{$\begin{array}{l}\text { POROSIDAD } \\
\text { POROSITY (\%) }\end{array}$} & $F_{14}$ & 35.0 & 21.0 & 17.0 \\
\hline & $F_{43}$ & 31.1 & 18.5 & 13.8 \\
\hline & $\mathrm{C}_{14}$ & 30.0 & 23.0 & 25.0 \\
\hline & $\mathrm{C}_{43}$ & 36.3 & 32.3 & 28.2 \\
\hline \multirow{4}{*}{$\begin{array}{c}\text { COMPRESIÓN } \\
\text { COMPRESSIVE } \\
\left(\mathrm{N} / \mathrm{mm}^{2}\right)\end{array}$} & $F_{14}$ & 26.3 & 37.3 & 35.5 \\
\hline & $F_{43}$ & 31.0 & 49.3 & 65.2 \\
\hline & $\mathrm{C}_{14}$ & 14.5 & 16.7 & 17.9 \\
\hline & $\mathrm{C}_{43}$ & 13.9 & 15.7 & 18.6 \\
\hline
\end{tabular}




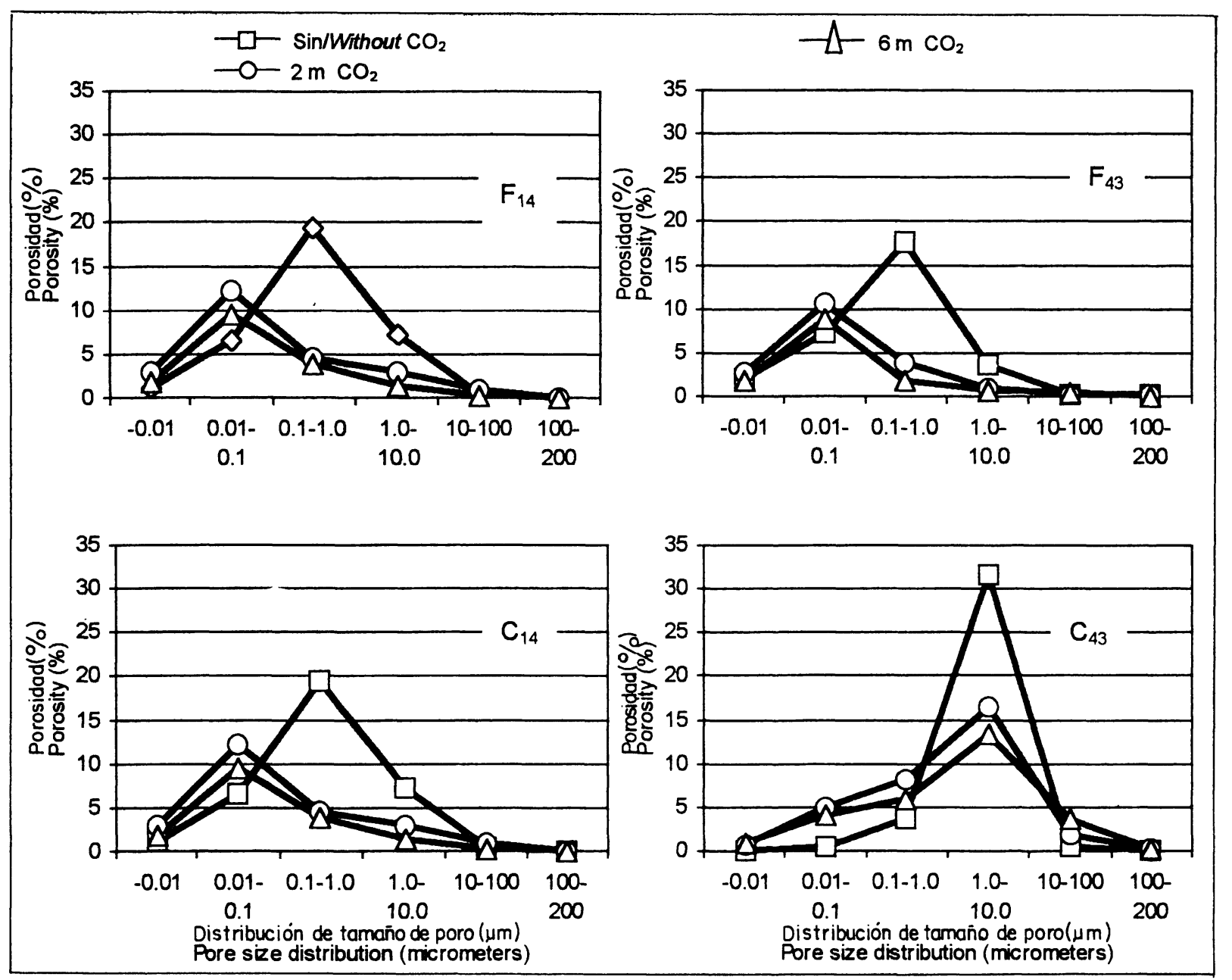

Figura 3.- Distribución del tamaño de poros.

Figure 3.- Pore size distribution.

\section{DISCUSIÓN}

El resultado de la reacción química entre el $\mathrm{CO}_{2}$ y las fases hidratadas del cemento de aluminato de cálcico es la formación de $\mathrm{CaCO}_{3}, \mathrm{AH}_{3}$ y $\mathrm{H}_{2} \mathrm{O}$. Las resistencias mecánicas se incrementan a medida que se produce la carbonatación debido al taponamiento de poros por los productos generados en esa carbonatación, lo que causá una disminución de la porosidad total y una variación en la distribución del tamaño de los poros; junto a esto no hay que descartar el carácter cementante del carbonato cálcico.

Los productos de carbonatación desarrollados son idénticos, independientemente del tiempo de curado y, además, las probetas presentan una composición homogénea, es decir, el exterior y el interior de éstas contienen los mismos compuestos y en proporciones relativas similares.

El carbonato cálcico formado como consecuencia de la reacción química de los hidratos de naturaleza hexagonal $\left(\mathrm{CAH}_{10}\right.$ y $\left._{2} \mathrm{AH}_{8}\right)$ y el $\mathrm{CO}_{2}$ fueron aragonito

\section{DISCUSSION}

The chemical reaction between $\mathrm{CO}_{2}$ and the hydrated phases of calcium aluminate cement results in the formation of $\mathrm{CaCO}_{3}, \mathrm{AH}_{3}$ and $\mathrm{H}_{2} \mathrm{O}$. Mechanical strength increases along with carbonation develops due to the clogging of the pores with products generated by carbonation, which causes the total porosity to diminish and modifies the pore size distribution; furthermore the cementitious character of calcium carbonate must not be neglected.

Carbonation products developed are identical no matter what the curing time is; moreover, samples present an homogeneous composition which means that inner and outer parts contain the same mineralogical composition in similar relative proportions.

Calcium carbonate formed as a consequence of the chemical reaction of hexagonal hydrates $\left(\mathrm{CAH}_{10}\right.$ and $\left.\mathrm{C}_{2} \mathrm{AH}_{\theta}\right)$ and $\mathrm{CO}_{2}$ were aragonite and vaterite. There 
y vaterita; la calcita no se produjo o se formó en muy pequeña proporción. La reacción de los hidratos de naturaleza cúbica $\left(\mathrm{C}_{3} \mathrm{AH}_{6}\right)$ da lugar a idénticas polimorfias del $\mathrm{CaCO}_{3}$, aragonito y vaterita. Es dificil establecer la polimorfia del $\mathrm{AH}_{3}$ como consecuencia de la carbonatación ya que se genera gran cantidad de gibbsita por la reacción química de hidratación.

Por ello puede establecerse una reacción general que exprese la carbonatación de los aluminatos cálcicos hidratados: was no formation of calcite or was indeed in very small proportion. Reaction of cubic hydrates $\left(\mathrm{C}_{\Im} A H_{\sigma}\right)$ produced identical polymorph of $\mathrm{CaCO}_{3}$, aragonite and vaterite. It is hard to establish the $\mathrm{AH}_{3}$ polymorph subsequent to carbonation, since the hydration process generates a large amount of gibbsite.

Therefore a general formulation can be established to express the carbonation reaction of the hydrated calcium aluminates:

$$
\left.\mathrm{C}_{\mathrm{x}} \mathrm{AH}_{\mathrm{y}}+\mathrm{xCO}_{2} \stackrel{75 \% \text { h. } \mathrm{r} .}{\longrightarrow} \mathrm{xCaCO}_{3} \text { (vaterita + aragonito) }+\mathrm{AH}_{3}+(\mathrm{y}-3) \mathrm{H}_{2} \mathrm{O}\right)
$$

En lo que se refiere a la velocidad de carbonatación fueron las probetas amasadas a $4^{\circ} \mathrm{C}$, constituidas por aluminatos cálcicos hidratados hexagonales, las más rápidamente carbonatadas. La mayor simetría cristalina de la forma cúbica frente a la hexagonal y la inestabilidad termodinámica que presentan los compuestos de naturaleza hexagonal justifica que estos sean más susceptibles a la acción del $\mathrm{CO}_{2}$. Dado que en la carbonatación de los aluminatos hexagonales se genera mayor proporción de agua que en el caso del $\mathrm{C}_{3} \mathrm{AH}_{6}$, puede disolverse el $\mathrm{CO}_{2}$ en mayor cantidad $\mathrm{y}$, por ello, la reacción progresa con más velocidad y más intensamente.

El mejor comportamiento mecánico se observó cuando el CAC se había hidratado a $4^{\circ} \mathrm{C}$. A la temperatura de $40^{\circ} \mathrm{C}$, probetas que presentaban un valor de porosidad del mismo orden que las amasadas a $4^{\circ} \mathrm{C}$; eran considerablemente menos resistentes. Debe tenerse en consideración, por tanto, que la porosidad total y las resistencias mecánicas de los $\mathrm{CAC}$, no están relacionados de forma sencilla, existiendo otros factores, como la distribución del tamaño de poros, que son de marcada influencia. Este hecho debe tenerse en cuenta a la hora de establecer ecuaciones para la resistencia mecánica. Por efecto de la carbonatación, las probetas experimentaron un descenso de la porosidad total (Fig. 4a) más marcado en las pastas preparadas a $4^{\circ} \mathrm{C}$ que a $40^{\circ} \mathrm{C}$. Este descenso en la porosidad se manifiesta en un incremento de las resistencias mecánicas (Fig. 4b). Este efecto se observa con más intensidad en las pastas curadas durante más tiempo. La causa de ambos fenómenos es la precipitación masiva de productos de carbonatación que se disponen, tanto en aquellos espacios ocupados inicialmente por los hidratos como en los poros ya existentes. Ello produce una disminución en la porosidad y un refinamiento en el tamaño del poro.

El tiempo de curado influye sobre el grado de carbonatacion y sobre la microestructura de las muestras, aunque no sobre su composición
As for the carbonation rate, samples mixed at $4{ }^{\circ} \mathrm{C}$, composed of hexagonal calcium aluminate hydrates, were those bearing faster carbonation. The better crystalline symmetry of the cubic compared to this of the hexagonal, and the thermodynamic instability shown by hexagonal compounds accounts for them to be more susceptible to the $\mathrm{CO}_{2}$ action. Since in carbonation of hexagonal aluminates a higher proportion of water is produced rather than in the case of the $\mathrm{C}_{3} \mathrm{AH}_{6}$, a larger amount of $\mathrm{CO}_{2}$ can get dissolved, thus, the reaction goes faster and is more intense in the first case than in the second one.

The best mechanical behavior was observed in $C A C$ hydrated at $4{ }^{\circ} \mathrm{C}$. Samples hydrated at $40^{\circ} \mathrm{C}$ having a porosity value of the same order than those mixed at $4{ }^{\circ} \mathrm{C}$ were remarkably less strong. Accordingly, the fact that total porosity and mechanical strength of the $C A C$ are not directly related and there are yet other factors as pore size distribution that have a strong influence. This fact must be taken into account when establishing equations for mechanical strength. Due to the carbonation effect, samples bore a more noticeable decrease of total porosity (Fig. 4a) in pastes prepared at $4{ }^{\circ} \mathrm{C}$ than in those prepared at $40{ }^{\circ} \mathrm{C}$. This porosity decrease results in the increase of mechanical strengths (Fig. 4b). This effect showed up with more intensity in pastes cured for a longer time. Both phenomena were caused by the massive precipitation of carbonation products that positioned themselves in the spaces initially occupied by hydrates, and in pores already existing. The result was a decrease of porosity and of the pore size fineness.

Curing time influences the carbonation degree and the microstructure of samples, although it has no effect on their mineralogical composition. Therefore, 

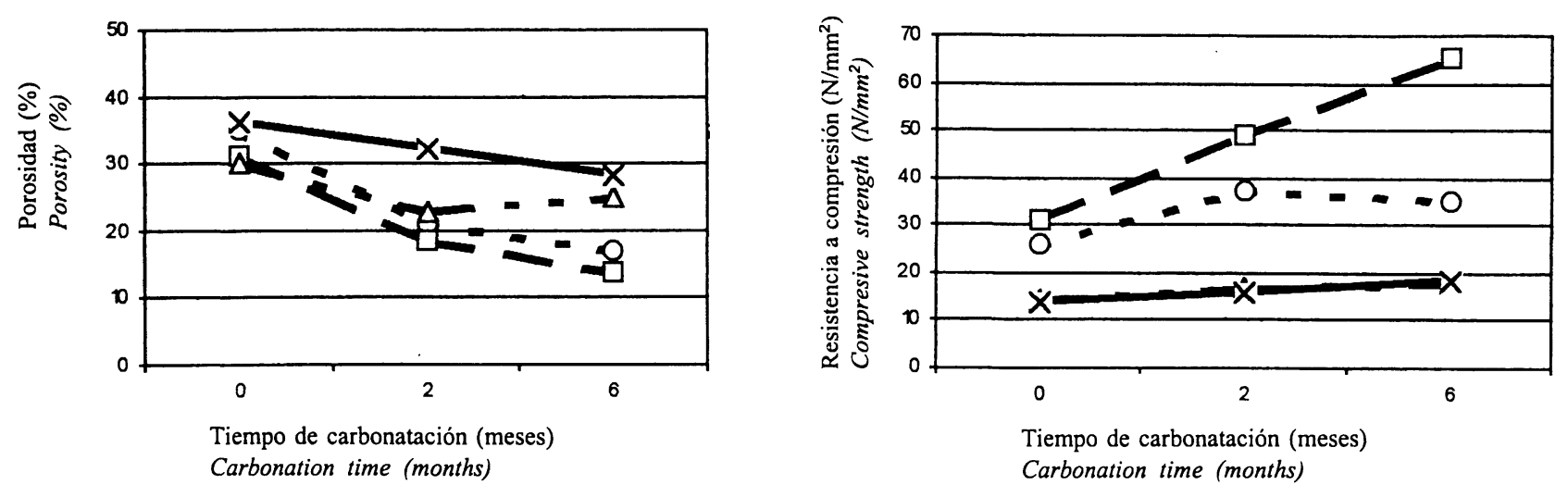

$$
-0-F_{14} \longrightarrow-F_{43} \longrightarrow-C_{14}-x-C_{43}
$$

Figura 4.- Evolución de la porosidad y las resistencias mecánicas con la carbonatación.

Figure 4.- Evolution of porosity and mechanical strength caused by carbonation.

mineralógica. Así, las muestras curadas durante un menor tiempo $\left(\mathrm{F}_{14}\right)$ son más porosas y menos resistentes que las correspondientes a un mayor tiempo de tratamiento $\left(\mathrm{F}_{43}\right)$. Tras la carbonatación, en ambas muestras disminuyó la porosidad total y aumentaron las resistencias mecánicas (Fig. 4), siendo el efecto más marcado en las pastas $\mathrm{F}_{43}$ cuyo grado de hidratación inicial era mayor. Se deduce, pues, la influencia decisiva de los métodos de curado (temperatura y tiempo) sobre las velocidades de carbonatación y microestructuras desarrolladas, las cuales, a su vez, evidencian modificaciones en los comportamientos mecánicos. De cualquier manera, los resultados obtenidos en el presente trabajo han evidenciado que como consecuencia del proceso de carbonatación en las condiciones realizadas, se produce un incremento en las resistencias mecánicas.

\section{CONCLUSIONES}

Las principales conclusiones que se pueden extraer del presente estudio son:

a) La velocidad de carbonatación de los aluminatos cálcicos de naturaleza hexagonal, $\mathrm{CAH}_{10} \mathrm{y} \mathrm{C}_{2} \mathrm{AH}_{8}$, es superior a la del aluminato cúbico, $\mathrm{C}_{3} \mathrm{AH}_{6}$.

b) El CAC hidratado evoluciona, como consecuencia de la acción del $\mathrm{CO}_{2}$, hacia la formación de carbonato cálcico en forma de vaterita y de aragonito, independientemente de si los aluminatos cálcicos hidratos originales son de naturaleza hexagonal o cúbica. $\mathrm{El} \mathrm{CaCO}_{3}$ (calcita) sólo se detecta a nivel de trazas. El hidróxido de aluminio que se forma al carbonatar las muestras elaboradas a $4^{\circ} \mathrm{C}$ es de naturaleza amorfa incluso transcurridos seis meses de carbonatación; cuando se detecta cristalino lo está en samples cured for a shorter time $\left(F_{14}\right)$ had more porosity and less strength than those cured for a longer time $\left(F_{43}\right)$. After carbonation, total porosity diminished in both samples and mechanical strength increased (Fig. 4). Pastes $F_{43}$ with higher initial hydration degree bore the strongest effect. The decisive influence of curing methods (temperature and time) on carbonation rate is thus proved and so is it the influence on the microstructures developed, which in turn evidenced behavioral mechanical changes. In any case results drawn from this work point out the increase of mechanical strength as a consequence of the carbonation process.

\section{CONCLUSIONS}

The outstanding conclusions drawn from this study are:

a) Carbonation rate of hexagonal calcium aluminates, $C A H_{10}$ and $C_{2} A H_{8}$, is higher than that of cubic aluminate, $\mathrm{C}_{3} A H_{6}$.

b) Hydrated CAC, as a consequence of the action of $\mathrm{CO}_{2}$, evolves towards a formation of calcium carbonate in the form of vaterite and aragonite, regardless the hydrated calcium aluminates are hexagonal or cubic. Only traces of $\mathrm{CaCO}_{3}$ (calcite) have been detected. The aluminum hydroxide formed during carbonation, in samples manufactured at 4 ${ }^{\circ} \mathrm{C}$, is found amorphous even after six months carbonation. When found crystalline it means it is as bayerite and norstrandite. The large proportion of 
forma de bayerita y norstrandita. La gran proporción de gibbsita formado por la hidratación del CAC a $40^{\circ} \mathrm{C}$ no permite determinar la polimorfia del $\mathrm{AH}_{3}$

específicamente generada en la carbonatación.

c) La resistencia mecánica de las pastas de CAC no presenta una relación lineal con la porosidad total. Otros factores tal como la distribución del tamaño de los poros son de gran influencia.

d) La temperatura y el tiempo de curado de las muestras no es un factor de importancia a la hora de influir sobre la naturaleza de los productos de carbonatación de las pastas de CAC, pero si influye en la microestructura desarrollada (porosidad) y en las resistencias mecánicas.

e) Los resultados obtenidos en el presente trabajo han evidenciado que, como consecuencia del proceso de carbonatación sobre pastas de CAC, en las condiciones realizadas, se produce un incremento en las resistencias mecánicas.

\section{AGRADECIMIENTOS}

Los autores agradecen a la Dirección General de Enseñanza Superior e Investigación Científica del Ministerio de Educación y Cultura español la subvención del proyecto PB97-1144, sin cuyos fondos no hubiese sido posible el presente trabajo. gibbsite formed as a consequence of the hydration of $\mathrm{CAC}$ at $40{ }^{\circ} \mathrm{C}$ does not allow determining the $\mathrm{AH}_{3}$ polymorph specifically generated during carbonation.

c) Mechanical strength of CAC pastes does not show a linear relation with total porosity. Other factors such as pore size distribution have an important influence.

d) Temperature and curing time of samples are not main factors regarding the degree of influence on the nature of carbonation products of CAC pastes, but in fact they have an influence on the resulting microstructure (porosity) and on the mechanical strength.

e) This work concludes that as a consequence of the carbonation process in CAC pastes, in said experimental conditions, an increase of the mechanical strength occur.

\section{ACKNOWLEDGEMENTS}

Grateful acknowledgements to Dirección General de Enseñanza Superior e Investigación Cientifica, of Ministerio de Educación y Cultura of Spain for financial support to this research through PB971144 project.

\section{BIBLIOGRAFÍA}

(1) Neville, A. M., "High alumina cement concrete", edited by Adam Neville in collaboration with P.J. Wainwright, England 1975.

(2) Fernández-Carrasco, L., "Procesos de hidratación y carbonatación del cemento de aluminato de calcio; influencia de los álcalis. Alteraciones microestructurales y relación con sus propiedades mecánicas".Tesis Doctoral, Universidad Autónoma de Madrid, Facultad de Ciencias, 2000.

(3) Midgley, H.G., Woodward, B.M., "The carbonation of high alumina cement".RILEM Int. S Symp. of Carbonation of Concrete, Wexham Springs, England 4/4, 5-6 april 1976.

(4) Sauman, Z., Lach, V., "Long -Term carbonation of the phases $3 \mathrm{CaO} \cdot \mathrm{Al}_{2} \mathrm{O}_{3} \cdot 6 \mathrm{H}_{2} \mathrm{O}$ and $3 \mathrm{CaO} \cdot \mathrm{Al}_{2} \mathrm{O}_{3} \cdot \mathrm{SiO}_{2} \cdot 4 \mathrm{H}_{2} \mathrm{O}$ ". Cem. Concr. Res. 2 , 1972,435-446.

(5) Raask, E., "The carbonation of high alumina cement concrete". RILEM Int. S Symp. of Carbonation of Concrete, Wexham Springs, England 4/4, 5-6 April 1976.

(6)Murat, M., Negro, A., Bachiorrini, A., "Modification de la cinetique d'hydratation de l'aluminate monocalcique en presence de carbonate de calcium". C.R. Acad. Sci. París 291, C, 1980, 287.

(7) Bachiorrini, A., Murat, M., "Evolution microestructurale des composites du systeme ciment alumineux granulat calcaire. I mode de propagation de la fisure", Cem. Concr. Res. Vol. 17, 242-248, 1987. 